\title{
NEGOCIOS FAMILIARES DE BARRIOS: DINÁMICAS SOCIOECONÓMICAS EN UN BARRIO DE TEMUCO, EN EL SUR DE CHILE
}

\author{
FAMILY-OWNED CONVENIENCE STORES: SOCIO-ECONOMIC \\ DYNAMICS IN A TEMUCO NEIGHBORHOOD, SOUTHERN CHILE
}

\section{EMPRESAS FAMILIARES: DINÂMICA SÓCIOECONÔMICA EM UM BAIRRO DE TEMUCO, NO SUL DO CHILE}

\section{Luis Guillermo Davinson Pacheco ${ }^{1}$ Ulises Armando Asenjo Ramírez ${ }^{2}$}

\begin{abstract}
Resumen
El artículo aborda los negocios familiares de barrios, a través de un estudio etnográfico, en el barrio Villa Los Creadores, del sector Fundo El Carmen de la ciudad de Temuco en el sur de Chile. En ese marco, su objetivo es profundizar sobre la creciente problematización que experimentan estas iniciativas, donde prima la subvaloración, los cambios en los patrones de consumo, los impactos socioeconómicos ante a una competencia desigual, el escaso apoyo gubernamental, y la invisibilización que tiende a manifestarse sobre estos comerciantes. Así a través de cuatro ejes, se profundiza sobre: vigencia; mecanismos que permiten su operación; las lógicas comerciales y los recursos tecnológicos. Los resultados y conclusiones constatan la validez y proyecciones de esta economía a pequeña escala; las formas de coexistencia que en el marco de la globalización asumen con la competencia; los patrones de convivencia vecinal en torno a la relación comercial y la generación de identidad barrial a partir del negocio familiar. También, aparece la multiplicidad de funciones de la familia en estas iniciativas, las modificaciones a los espacios físicos y el uso de Redes Sociales como mecanismo publicitario.
\end{abstract}

Palabras clave: Microempresas, emprendimientos, familias, menudeo.

Doi: https://doi.org/10.15359/eys.25-58.5

Recibido: 21-07-2020. Reenvíos: 07-10-2020. Aceptado: 05-11-2020. Publicado: 06-12-2020.

1 Doctorante en Ciencias Sociales en estudios territoriales Universidad de Los Lagos, Chile/ Universidad de La Frontera, Chile. Correo electrónico: guillermo.davinson@ufrontera.cl ORCID: https://orcid. org/0000-0002-2850-891X

2 Magister en Gerencia Social, Universidad Tecnológica INACAP Temuco, Chile. Correo electrónico: ulises. asenjo@inacapmail.cl ORCID: https://orcid.org/0000-0001-9609-1370

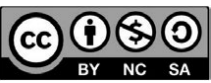




\begin{abstract}
Family-owned convenience stores are addressed through an ethnographic study in Villa Los Creadores, a neighborhood in Fundo El Carmen, Temuco city, southern Chile. The objective is to take a closer look at the growing problems experienced by these initiatives, including undervaluation, changes in consumption patterns, socio-economic impact due to unequal competition, lack of government support, and the frequent invisibility of merchants. Consequently, using four axes, the paper is focused on validity, the mechanisms that allow operations, commercial logic, and technological resources. Results and conclusions confirm the validity and projections of this small-scale economy, the ways of coexistence they assume with the competition in the framework of globalization, the patterns of neighborhood coexistence around the commercial relationship, and the generation of neighborhood identity of family-owned stores. The multiple functions of the family, modifications to physical spaces, and the use of social networks as an advertising mechanism also appear in these initiatives.
\end{abstract}

Keywords: microenterprises, ventures, families, retail

\title{
Resumo
}

O artigo trata das empresas familiares por meio de um estudo etnográfico no bairro Villa Los Creadores, no setor Fundo El Carmen da cidade de Temuco, no sul do Chile. Neste quadro, pretende-se aprofundar na crescente problematização vivida por estas iniciativas, onde prevalece a subvalorização, as mudanças nos padrões de consumo, os impactos socioeconómicos face à concorrência desigual, o escasso apoio governamental e a invisibilidade que tende a se manifestar sobre esses comerciantes. Assim, por meio de quatro eixos, aprofunda-se em: validade, mecanismos que permitem seu funcionamento, lógicas comerciais e recursos tecnológicos. Os resultados e conclusões confirmam a validade e as projeções desta economia de pequena escala; as formas de coexistência que, no quadro da globalização, assumem com a concorrência; os padrões de convivência de vizinhança em torno da relação comercial e a geração de identidade do bairro a partir da empresa familiar. Além disso, a multiplicidade de funções familiares aparece nessas iniciativas, nas modificações nos espaços físicos e no uso das redes sociais como mecanismo de publicidade.

Palavras-chave: microempresas, emprendimentos, famílias, varejo.

\section{INTRODUCCIÓN}

Para la realidad latinoamericana, el rol que juega en la cotidianidad de cualquier barrio, un negocio del sector ha sido poco relevado. Así, pulperías, boliches, changarros, ventorrillos; o almacén, son expresiones caracterizadas por la venta al menudeo y punto social del 
encuentro vecinal. En este caso los negocios del barrio, de propiedad y administración familiar son las unidades por analizar, inscribiéndose para la realidad chilena como muestra de una dinámica habitual de la vida comunitaria. Lo anterior, se inscribe en la perspectiva antropológica, donde las relaciones económicas, son sustanciales a la vida material y sociocultural humana. Así dos enfoques paradigmáticos: Mauss (2009) con el intercambio y circulación de bienes como origen de la vida social, no por el valor cultural de los bienes, sino por las relaciones y vínculos sociales emanados del intercambio. A su vez, Malinowski (2001): el valor a los objetos intercambiados es subsidiario del valor económico, del parentesco y de reconocimiento que se producen inter-comunidades mediante el intercambio. Ambos enfoques, nos alertan de las motivaciones, que muchas veces trascienden lo estrictamente económico y realzan los aspectos sociales.

El objetivo del artículo apunta a desarrollar la problemática asociada a que en la última década se habrían producido impactos económicos y sociales producto de la competencia con las grandes cadenas comerciales; y cambios en los patrones de consumo del vecindario, pese a ello, estas iniciativas no se han subvalorado en los barrios. Fuera de este entorno, prima invisibilización y desconocimiento de sus particularidades, que se agrava por la escasa preocupación gubernamental y sistematización técnica sobre estos emprendimientos produciendo un marco complejo de anonimato comercial y augurios de desaparición.

Como segundo punto, una revisión empírica en el barrio Villa Los Creadores, del Sector Fundo El Carmen de la ciudad de Temuco, en el sur de Chile, permite abordar los negocios de barrios, de propiedad y administración familiar. A partir de su naturaleza fundacional, el acuerdo familiar y comercial, proporcionan insumos para conocer los mecanismos internos subyacentes en este tipo de emprendimientos. En una cultura capitalista, promotora de la competencia y responsabilización individual, resulta interesante la existencia de fórmulas internas familiares, que permiten su funcionamiento. En tercer lugar, la descripción de las lógicas internas, posibilitan la descripción de uniformidades en los negocios del barrio; y de la utilización de algunas herramientas tecnológicas, que en contextos vecinales están siendo utilizadas por estos negocios para mantenerse vigentes.

El Gobierno a través de un organismo para las micro y pequeñas empresas, denominado Servicio de Cooperación Técnica (SERCOTEC) los define como "aquel pequeño comercio minorista que provee de diversos artículos, especialmente abarrotes a los habitantes y transeúntes de un barrio residencial y/o comercial". Actualmente, estos negocios enfrentan al comercio de las grandes cadenas de tiendas y supermercados, que proyectan imagen de comercio moderno, que hace "mucho marketing, convoca mucho público y es más visible" (Gálvez, et al. 2009, p. 10); disímiles a las connotaciones que representan los negocios de barrios, de ventas a pequeña escala, con atención cordial y personalizada.

El artículo se estructura, posterior a esta introducción, abordando los aspectos metodológicos, los resultados (con tres secciones referidas a los mecanismos internos; lógicas y dinámicas comerciales y nuevas tecnologías); y por último, las conclusiones. Aspectos que esperamos contribuyan a visibilizar y reconocer el aporte de las familias que mantienen vivas estas iniciativas pese a la competencia de mega comercios y los 
cambios en los patrones de consumo. Así, destacamos estos sistemas colaborativos, priorizando la convivencia vecinal y contribuyendo a satisfacer necesidades y generando identidad cultural en el sector.

\section{ASPECTOS METODOLÓGICOS}

\subsection{Instrumentos y trabajo de campo}

La investigación se desarrolló a través del método etnográfico y se inscribió en el contexto de un trabajo de grado del Programa de Magister en Gerencia Social de la Universidad de La Frontera, Temuco, Chile. Dentro de sus objetivos, destacaba para nuestros fines, el desarrollo de un diagnostico económico, comercial y tecnológico, aplicado a los negocios familiares del barrio Villa Los Creadores del sector Fundo El Carmen de Temuco. Respecto a los sujetos participantes se concentró en 45 iniciativas comerciales, asociadas a patentes de la Municipalidad de Temuco. Cumpliendo con la característica de ser Microempresa Familiar (MEF) que la Ley º 19.749 / 2001 establece.

La Patente Comercial en este caso, corresponde al territorio comunal de la Municipalidad de Temuco, organismo que, a través de la Oficina de Rentas y Patentes, entregó la base de datos, correspondiente al Rol vigente para el 2019 donde se registraban, estas 45 patentes comerciales, abarcándose en el estudio el $100 \%$ de las MEF identificadas con patente vigente. En lo referido al proceso de recolección de datos, se organizó un equipo de 10 investigadores de campo, estudiantes de la Carrera de Ingeniería en Administración de Empresas, de la Universidad Tecnológica INACAP de Temuco. Este equipo se encontraba cursando la Asignatura de Fundamento de la Economía, siendo responsable el profesor Ulises Asenjo Ramírez y se realizó la definición de los locales a través de la geo referenciación.

La observación participante, se realizó durante octubre y noviembre de 2019. Respecto de los instrumentos de recolección de información, destacó la aplicación de entrevistas semi estructuradas, las cuales se componían de cuatro secciones; a) identificación; b) ámbitos económicos, comerciales y sociales; c) desarrollo tecnológico y; c) recursos humanos y capacitación.

\subsection{Contexto Etnográfico}

Villa Los Creadores, es un barrio fundado el 4 de noviembre de 1998, donde unas 325 familias ocuparon dicho sector. En noviembre del 2009, según registros del Diario Austral de Temuco refería, que: "contaba con un microcentro de salud, dos complejos educacionales, un supermercado, dos salas cuna y jardines infantiles, organismos que han facilitado la vida de las familias". Al permitir acceso a servicios en el barrio, sin trasladarse al centro de la ciudad, contribuyó a fortalecer pertenencia al barrio, que se encuentra en el macro sector de Fundo El Carmen, ubicado a varios kilometros del centro cívico de Temuco, alcanzando una población de $\mathbf{5 0 . 0 0 0}$ habitantes. En la actualidad, sirve como espacio residencial y en el último lustro, se han desarrollado programas de equipamientos gubernamentales, para mitigar la segregación en relación al acceso a servicios urbanos. 
La conectividad vial se presenta en forma precaria, dado que los accesos, son estrechos para el parque automotriz y los residentes. La distancia aproximada al centro de Temuco es de unos siete kilómetros. En la actualidad, según calculos del Centro de Salud Familiar del sector, residen en este barrio unas 1200 personas. Este sector a dos decadas, cuenta con identidad barrial, y donde un elemento de su dinamica citadina, son justamente este tipo de emprendientos familiares, que exceden lo meramente comercial y son reflejo de solidos puntos de encuentro, red de colaboración, recreación y confianza vecinal.

\section{ALGUNOS RESULTADOS: MECANISMOS, LÓGICAS Y TECNOLOGIAS}

\subsection{Mecanismos internos}

En este caso estamos describiendo las dinámicas de un "negocio del barrio", concepto que acentúa la cualidad de pequeño, dado que se trata de un espacio con dimensiones equivalentes a un $1 / 4$ de una vivienda social, de las que construye el Servicio de Vivienda y Urbanismo, de $45 \mathrm{~m}^{2}$ y cuenta con productos de primera necesidad, los más demandados por la clientela del barrio.

Un marco explicativo apunta a cuatro elementos que determinan la vigencia de estos negocios del barrio, con propiedad y administración familiar y venta al menudeo y la fórmula conceptual de propietarios/familiares, nos refiere a esta dualidad. En primer término: el parentesco; luego, la unión de lo doméstico y productivo; en tercer lugar: multifuncionalidad de tareas y, por último: solidaridad vecinal.

Parentesco, entendido como un vínculo biológico o por afinidad que, en este contexto, actúa como: el primer recurso de mano de obra y; ente aglutinador sobre el objetivo comercial, constituyéndose una plataforma fundamental de actividades económicas en vastas comunidades de América Latina. Se ha planteado que la globalización, por su carácter impositivo y homogenizante, como muchos auguraban, generaría mayor fragmentación parental, augurio que ha quedado seriamente cuestionado a partir de la proliferación de los negocios familiares y su mantención en espacios globalitarios.

La expresión material del rol del parentesco se expresa en el desarrollo de determinados trabajos considerando la existencia de una cadena de aportes familiares; tanto quienes viven en el hogar y también los de fuera de éste. El parentesco asegura la supervivencia de los actores sociales "depende de su capacidad para crear un sistema de intercambio completamente diferente de las reglas de mercado" (Lomnitz, 1994, p. 92). La importancia del parentesco en las sociedades capitalistas contemporáneas está demostrada desde los estudios antropológicos de los 70, donde muchos "proyectos económicos se basan en tales parentelas entrelazadas, sobre todo entre hermanos" (Rothstein, 2007, p. 155).

Fusión (unión) espacial doméstica y productiva: Una de las características en estos negocios familiares de barrios, lo constituye la transformación física del inmueble, donde una parte es destinada como local de venta y que suelen estar adosado a la casa habitación de la familia propietaria del negocio. Dicha transformación, no es tan solo física, sino que también es social y tiene implicancias dado que el hogar se transforma

Luis Guillermo Davinson Pacheco - Ulises Armando Asenjo Ramírez

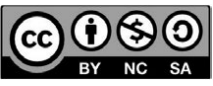


en espacio dual, doméstico y productivo respectivamente. En estos casos, el hogar por un lado posibilita la vida familiar y por otro, simultáneamente el desarrollo comercial, fusionándose en muchos momentos ambos planos, y posibilitando, que operen yuxtaposiciones, y se naturalicen las más diversas actividades: encargos, los reemplazos, seguridad, aseo, educación de los hijos y diversas otras tareas durante parte de los ciclos vitales de sus integrantes están permeadas en esta relación con el entorno de la compra y venta.

Multifuncionalidad de reemplazos: En muchos casos sí los integrantes del grupo doméstico; incluidos niños y ancianos asumen con un definido marco de funciones productivas a ser ejecutadas, permite una versatilidad individual como también del conjunto de individuos para desplazarse ocasional o simultáneamente a otra actividad productiva. Especialización que permite a un mismo sujeto pueda a desarrollar varias labores durante el ciclo doméstico. Adquisiciones, limpieza y seguridad por nombrar algunas; dando continuidad comercial. En los contextos rurales: labores agrícolas, artesanales, comerciales, y migración temporal son desarrolladas en esa línea. Los principales encargados de materializar esta cadena de reemplazos son el grupo residencial, ocasionalmente otros familiares, fortaleciendo el grupo y proyectando la actividad económica.

Solidaridad vecinal: Las relaciones de cooperación en los barrios responden a distintos patrones motivacionales. En ese sentido, la búsqueda de un sustrato de apoyo que posibilita la sobrevivencia de los grupos a partir de las reciprocidades entre vecinos que no tienen entre sí lazos consanguíneos, sino más bien en relaciones de amistad, en vínculos comunitarios, que se expresan en acciones colectivas. En la relación comercial vecinal, surgen episodios de favores que son retribuidos de distintas maneras; relaciones sociales fundamentadas en la confianza, suponiendo cierto grado de conocimiento y familiaridad entre las personas que la entablan; proximidades sociales, físicas y económicas que les permiten mantener relaciones de reciprocidad mutua. En este barrio con una historia de poco más de 20 años, estos vínculos comunitarios se refuerzan, por una historia de asentamiento compartida y una construcción de redes sociales recientes.

Estos cuatro elementos explicativos, parentesco, fusión doméstico-productiva; multifuncionalidad y solidaridad son aspectos que posibilitan la vigencia y consolidación del negocio familiar de barrio. En lo especifico, un análisis de carácter descriptivo, a través de ciertos ejes, permite conocer algunos aspectos cotidianos y operativos de estos negocios familiares del barrio. Estos emprendimientos, en la jerga vecinal son conocidos como "almacenes" o "negocios" y sus propietarios/familiares los nominan con diversos nombres que muchas veces aluden a sus propietarios o especificidad. En estas lógicas y dinámicas comerciales, aparecen decisiones familiares; motivaciones; modificaciones a la vivienda; horarios; publicidad; pago de patentes, y un ámbito de tecnologías y redes sociales. 


\subsection{Lógicas y dinámicas comerciales}

\subsubsection{Decisiones familiares}

Al remontarse los propietarios/familiares a sus inicios, aflora un consenso respecto a fijar esos momentos como trascendentales, en términos de expectativas e incertidumbre respecto al desafío que acometerían. Un segundo elemento, fueron los largos procesos de análisis, previo a la toma de decisiones, que bajo la figura de "hacer averiguaciones con amigos, familiares y conocidos, con experiencias en actividades comerciales", realizaron como familia, para asegurarse del éxito de la iniciativa. Esta suerte de estudio de mercado, fueron elemento contribuyente a la decisión. Como tercer aspecto, se refiere a que el impulsor, es quien en los hechos posteriormente aparece ante la institucionalidad como responsable del negocio. El emprendimiento conlleva una decisión familiar, que en algunos negocios de este tipo comprende el trabajo de todo o parte importante del grupo doméstico.

\subsubsection{Aspectos motivacionales}

Al definir la motivación como impulso inicial que genera la fuerza necesaria para iniciar una acción comercial, con la capacidad persuasiva para seducir a otros integrantes del grupo familiar, logrando su adherencia. Al respecto, la motivación recurrente y generalizada, que aparece en este estudio, fue la necesidad económica del sujeto impulsor de la iniciativa. La búsqueda de ingresos económicos suficientes para satisfacer las necesidades familiares fue el contexto para asumir con estos desafíos familiares, como alternativa viable para generar dichos ingresos. Un segundo aspecto motivacional es lo definido como sentido de independencia, traducido en la resistencia a experimentar sometimiento a eventuales jefaturas de terceros. Quienes fueron empleados dependientes, valoran especialmente esta autonomía e independencia que dicen obtener en estos negocios.

Además, es percibido como una actividad no tan sacrificada, dado que no depende de horarios impuestos y de subordinación. Como tercer ámbito se inscribe el sentido de oportunidad, dado que el poblamiento de este barrio fue por etapas y con ello, se generaron micro sectores del Barrio, que no contaban con negocios. Esto último motivaba a instalarse, para cubrir las necesidades vecinales de consumo, por tanto, se vislumbró como una oportunidad de negocio, al mismo tiempo que se resolvía la necesidad de acceso a provisiones de los vecinos.

\subsubsection{Modificaciones estructurales}

La fusión de los espacios domésticos y productivos traduce en los hechos la fusión de familia y trabajo. El hecho que sea el hogar y la vivienda su expresión material da coherencia a un negocio familiar en términos empíricos. Así entonces, la necesidad de habilitar la casa de la familia, con la finalidad de contar con un espacio para la instalación del negocio, obligó imperiosamente en la gran mayoría, a alterar la obra original y 
hacerle modificaciones estructurales, muchas de ellas, de envergadura de una vivienda de $45 \mathrm{mts}^{2}$.

Se refiere entre los propietarios/familiares a estas modificaciones como: "ampliaciones" o "agregados". Esta lógica de agrandar la casa, junto con ser expresión material del cambio, es también referencia a una modificación en el estatus de la familia, que pasan a ser los vecinos del negocio. Estas alteraciones a la obra principal suelen hacerse en los espacios laterales, diseñados originalmente para aparcar vehículos; o bien en el frontis de la vivienda, en lo que se denomina el antejardín y donde se procede a construir una ampliación; sección que implica acercar la pared de la casa al cerco perimetral y por ende la vía pública.

Como resultado de estas intervenciones en la infraestructura de la vivienda familiar, es posible advertir cambios en ventanales, puertas, habilitación de garajes y antejardines. También es posible observar, que, en aquellas viviendas de dos pisos, dividen el espacio y dejan el primer piso para negocio. Al no contar con espacios en la vivienda, para ser utilizadas como bodegas, es usual el apilamiento de diversos enseres en los antejardines y espacios posteriores, primando los más diversos artículos o implementos y material en desuso.

\subsubsection{Horarios de atención y volúmenes de compras}

La gestión y operación comercial, se organiza en horarios definidos para la atención de público. Primando distribución en jornadas con tiempos establecidos y publicados. Ello, implica que, no existe atención en horario continuado, ya que los comerciantes no escapan a las lógicas familiares y muchos cierran durante el almuerzo, generalmente de 13.30 a 14.30 horas. Sin embargo, los vecinos respetan estas normativas no obstante saben que tienen el privilegio dado que son clientes permanentes, de concurrir a comprar, "tocar el timbre" y con ello ser atendidos independientemente cierre de horario; situación similar que sucede en la tarde noche, ya posterior a las 20.00 horas. Lo anterior, en algunos casos es una opción ofrecida como valor agregado al negocio: "Si está cerrado y la luz prendida, toque el timbre".

Por otro lado, los proveedores concurren a abastecer y proceden a estacionar el vehículo de transporte de carga en la vía pública, frente al negocio, para así descargar los más diversos elementos y mercaderías, acción que se realiza generalmente trasladando los productos "a mano" y en ocasiones debe utilizarse un carro pequeño de arrastre manual, en una "yeguita" (expresión local para estos carros de 2 ruedas) lo que revela el volumen de productos que se manejan en este tipo de negocios de barrios.

En cambio, en el caso de los clientes, estos concurren a comprar y no requieren de carros para trasladar sus adquisiciones dado que los volúmenes adquiridos son reducidos en tamaños y pesos, y por ende transportables en bolsas o alguna mochila, dado que concurren sin vehículos, por la cercanía negocio clientela, que es una característica inherente a este tipo de negocio. El arribo al lugar es siempre en forma peatonal, y 
tampoco estos negocios cuentan con estacionamiento y de ser el caso el vehículo debe estacionar en la vía publica cerca del establecimiento.

\subsubsection{Mixtura publicitaria}

La necesidad de comunicar a los clientes respecto de promociones, de los productos comercializados y del nombre de los locales, obliga a una fusión amplia de múltiples avisos que varían en la forma y en el fondo; sin que existan evidencias de alguna normativa que regule esta materia. Así, en el barrio en estudio, las fachadas de los locales comerciales son espacio para instalar la mayor cantidad de publicidad, que, sin mucha organización, lo que genera una imagen de relativo desorden. Se fusiona esta publicidad, de tipo estandarizada y de mejor calidad, (proporcionada por las grandes empresas, con fines publicitarios de sus marcas) con la que el propio comerciante utiliza para difundir otros productos, esto último a través de pizarras o letreros de cartón, misma que se puede instalar en las rejas de las casas negocios.

Las cadenas de empresas nacionales entregan letreros y otros productos de publicidad estandarizada, y como tal, dependiendo de las prácticas del Marketing de estos grandes proveedores, los negocios del barrio estarán abarrotados de banderas, afiches, banderines y pancartas. En el ámbito de la publicidad aparece como un medio recurrente, el avisaje por radio, donde destaca, una radioemisora, Mirador de Temuco, (con oficinas en el centro de esta ciudad) y se caracteriza por tener contactos directos e interactivos con sus oyentes, publicitando negocios, ofertas y promociones. También existe publicidad en la vía pública u otros espacios, es así como los letreros, tipo palomas, son ubicados en lugares estratégicos del barrio: plaza, paraderos de microbuses, y calles principales.

Es posible, encontrar pizarras escritas con tiza (localmente llamadas "palomas" que son transportables, pero con atril y doble cara) en el exterior, promocionando la existencia de productos sin los precios de estos. Además, es común, afiches que proveen las grandes Empresas Embotelladoras promocionando gaseosas, agua mineral y cervezas, en los frontis y ventanas de estos establecimientos. Por otro lado, las banderas de empresas de productos de Cecinas, Confitería, Helados o la popular Coca Cola, sumados a letreros de empresas de Telefonía Móvil generan un escenario multicolor e imposible de pasar desapercibido. Entre los rubros de nuevos productos, que mantienen vigencia y son ofrecidos en estos negocios de barrio, se encuentran los insumos y materiales para uso de computadoras, impresiones, fotocopias y arriendo de consolas de juegos electrónicos; junto con chip de prepago de telefonía móvil, audífonos y otros, de especial demanda entre los clientes más jóvenes; algunos locales también incluyen algunos artículos de librería demandados por los escolares.

\subsubsection{Rubros y Patentes Comerciales}

La elección del rubro comercial resulta importante en este tipo de emprendimientos económicos, porque deja de manifiesto, el volumen del capital inicial necesario para estos fines; así como también las exigencias en cuanto a normas sanitarias u otras que deben cumplirse según sea el giro del negocio. En ese marco, aparecen tres ámbitos 
generales: el primero referido a aquellos que comercializan abarrotes básicos y el segundo refiere a todos los vinculados a elaboración de "comida rápida" o "al paso"; y que en términos populares sindicarían como "comida chatarra". Priman, en estos, la elaboración de completos (Hot dog); además de sándwich y venta de papas fritas, gaseosas, café instantáneo y algunos snacks, cuestiones que son al menudeo. También lo que acá lleva por nombre como "salchipapas" (salchichas con papas fritas con algún aderezo). La excepción a esta categorización de chatarra, entre locales que venden comida preparada, estaría dada por los negocios de venta de Sushi. Como tercer ámbito, aparecen librería, que se traduce venta de cuadernos, lápices, pegamentos, cartulinas, también uno que tiene que ver con una maquina multifuncional, para realizar impresiones y fotocopiado. Estos ámbitos, dada su naturaleza es posible sindicarlos como de escasa complejidad comercial y bajo volumen de capital inicial. Sin embargo, la complejidad en el manejo de este tipo de negocios estaría dado por: la atención del público y la relación con los proveedores.

Respecto de las patentes, hay que señalar que, por razones normativas, estos negocios deben contar con Patente Comercial, y como tales su clasificación en la Corporación Edilicia es de Microempresa Familiar, debiendo cumplir con dicha caracterización, y con el hecho que desarrollan giros no contaminantes y su ubicación es una casa habitación, como lo establece la normativa. Además, en los casos que expendan alcoholes, deben contar con esa categoría de patente. Especial mención en el caso de preparación de alimentos, es la tramitación de resolución sanitaria que les habilita, lo que muchas veces implica adecuaciones a la infraestructura e inversión en equipamiento, además de estar sometidos a fiscalizaciones de la autoridad de salud.

\subsection{Nuevas tecnologías}

\subsubsection{Percepción tecnológica}

Los propietarios/familiares manifiestan valoración a la tecnología, entendida para estos efectos, como el conjunto de artefactos automatizados y energizados a través de la red de electricidad y son reconocidos, como de uso habitual, las balanzas, congeladores, vitrinas de frio, cortadoras de cecinas, máquinas de venta de café, microondas y hornos eléctricos. Sin embargo, asocian a un alto costo, todo lo que implique implementación tecnológica, de apoyo al proceso de venta, y, por ende, minimizan su necesidad evitando el adquirir este tipo de recursos.

Por ello, sumado al hecho de no contar con recursos económicos disponibles, no incursionan en este tipo de inversiones, a no ser que sea a través de financiamiento proveniente proyectos de fomento emanados del aparato estatal. El conocimiento sobre los recursos tecnológicos también es una variable deficitaria, y el teléfono móvil inteligente, es el más valorado, dado que es el preferentemente utilizado como medio para acceder a internet. Lo anterior, implica que en general, no cuentan con equipos computacionales en el negocio propiamente tal, ni tampoco Software que permita contribuir en la gestión del negocio. 
Donde existe coincidencia es lo imprescindible del uso de Transbank (empresa monopólica de administración de transacciones con "dinero plástico" o tarjetas) que permite el pago con tarjetas de débito o crédito, dada la proliferación del uso de los plásticos entre sus clientes y como tales, han debido adaptarse a esta forma de pago para no perder clientela; lo que también es un factor protector, ya que al no existir en caja dinero en efectivo, se evita la delincuencia.

\subsubsection{Venta y promoción en redes sociales}

La proliferación en la sociedad chilena, en el último decenio, de las Redes Sociales, instaura una práctica social de comunicación por estos medios, donde los emprendedores familiares, no escapan a estas lógicas comunicacionales. Así, señalan, que publicitan a través de la plataforma de Facebook, a través del perfil de Fundo El Carmen, que es una cuenta abierta para todos los pobladores de este sector. Proliferan en este medio, las ofertas, promociones y cambios de los horarios de atención. Altamente valorado y referido como "de gran utilidad", dado que no implica costos para acceder a este medio. A este tipo de plataforma se suman algún otro tipo de recursos circunstanciales, pero son los hijos/as o nietos/as de estos comerciantes, quienes administran este recurso, dado el escaso dominio tecnológico de los adultos, por la brecha generacional existente en esta materia.

\subsubsection{Plataformas Tecnológicas del Estado}

El Gobierno ha realizado un esfuerzo tecnológico por implementar sitios virtuales de uso ciudadano y optimizar procesos que avancen hacia un modelo de Gobierno Electrónico. Sin embargo, estos propietarios/familiares, delegan en sus Contadores, este tipo de vinculación, y son estos profesionales quienes acceden a la plataforma del Servicio de Impuestos Internos (SII) para gestionar pago del impuesto mensual IVA, y otros. También estos profesionales usan PREVIRED, servicio de declaración y pago de cotizaciones previsionales por Internet, para los trabajadores dependientes.

En otros trámites recurrentes, en lo que compete al uso de plataformas web, del Servicio de Registro Civil (SRC), permite trámites valorados, por cuanto impide abandonar el negocio, para acudir a las oficinas, considerando que es asumido como un problema, ya que, al cerrar para ir a hacer algún trámite, implica pérdidas en ventas y molestia para los clientes. Aparece también de uso frecuente, la plataforma del Banco Estado (nombre comercial del Banco del Estado de Chile) para consultar estado de cuenta, saldos, cartolas, etc. Incluso ha aparecido como servicio adicional en algunos de los negocios de barrio, la llamada "Caja Vecina" del Banco Estado, que permite realizar algunas transacciones bancarias básicas como giros o depósitos, por montos acotados; lo que también facilita este tipo de trámites a los vecinos, en barrios donde no existen cerca sucursales bancarias o cajeros automáticos. 


\subsection{4 Ámbito de la Capacitación}

Se constata que los comerciantes reconocen que existe un déficit de Capacitación en su rubro, y reconocen también, que es necesario capacitarse, aunque señalan que deben ser instancias prácticas y acotadas en el tiempo, al ser implementadas las capacitaciones, no pueden ser extensas, dado que el factor tiempo, es un recurso muy importante en las dinámicas cotidianas del negocio. Una externalidad interesante de considerar al planificar capacitaciones consiste en tener presente la percepción, de que la capacitación podría ser compleja para ellos, dado que estos comerciantes entrevistados, en su mayoría, refieren haber transcurrido muchos años de haber egresado del sistema escolar y no cuentan con especialización teórica en el rubro comercial. Por otro lado, al consultar sobre las áreas de interés, sus demandas guardan relación con ventas, marketing, administración y computación. Interesante resulta percibir la asociación que se suscita entre nivel de escolaridad o educación formal y capacitación, en tanto existencia de herramientas o capacidades personales para capacitarse. Los mecanismos internos, las lógicas de operación y el uso de tecnología, constituyen una triada importante a ser destacada en estos negocios familiares de barrios. Sin embargo, el parentesco, la fusión domestica-productiva, los reemplazos y la solidaridad vecinal conforman el sustrato de su vigencia.

\section{CONCLUSIONES}

Se constata la plena vigencia y consolidación de estas iniciativas; como también el aporte que realizan en tanto actores comerciales, pero también sociales, dinamizando con ello, la cultura y vida cotidiana del barrio. Pese a los cambios en los hábitos de consumo de los vecinos, la penetración de las grandes cadenas y la escasa preocupación gubernamental por este sector; estos negocios familiares siguen siendo referentes identitarios del barrio. En la praxis, son prueba fehaciente a la falacia del Chile emergente, que no asume la existencia de indicadores de cerca de un $50 \%$ de su población, sobrevive gracias a la economía a pequeña escala, informal en todas sus combinaciones y los negocios al menudeo como que hemos analizados.

La competencia y en ocasiones la agresividad comercial que impone el modelo no ha logrado en la práctica permear con esas actitudes los negocios familiares del barrio, que presentan un patrón de razonamiento colaborativo con el entorno donde se emplazan, que junto con satisfacer las necesidades familiares, sus estrategias comerciales consideran la convivencia vecinal, primando en ocasiones, la categoría vecino más significativamente que la de cliente y por ello, son valorados por el vecindario. El anonimato que impera en las transacciones de los grandes negocios de cadenas del centro de la ciudad es contrarrestado con este modelo de negocio tradicional, caracterizado por su rapidez, cercanía, y lugar de convivencia e interacción social de los que acuden.

Los negocios familiares del barrio Villa Los Creadores; en estos 20 años, no habían sus experiencias e historias sistematizadas, generando en las percepciones de los afuerinos, indicios de invisibilización, anonimato y escaso aporte a la vida comunitaria por parte de estos emprendimientos; pero otra es la sensibilidad que para sus vecinos reviste, dado 
que son calificados como vitales para satisfacer necesidades inmediatas, pero también valorados como micro espacios de identidad barrial, y contribuyentes como reforzadores de vínculos comunitarios de solidaridad vecinal.

El excedente a lo estrictamente comercial no es registrado en los indicadores oficiales; de ahí la necesidad de dar a conocer su vigencia, en tanto siguen siendo puntos de socialización más allá de lo comercial, que; sirven a organizaciones comunitarias festividades y eventos deportivos locales. Funcionalmente, es usado para encargar algún objeto; o dejar avisos en búsqueda y ofrecimiento de trabajo del vecindario; también, referente de seguridad barrial, donde es posible dejar las llaves al almacenero en ausencia del residente; recibir encargos e incluso ser lugar de espera para los niños a la hora del arribo del transporte escolar. La presencia de este tipo de acciones por parte de estos negocios es prueba de una vigencia sólida y creciente de reciprocidades en los dispositivos comunitarios donde se encuentran emplazados.

Algunos de los aspectos identificados, pudiendo haber otros, que guardan relación con la vigencia y consolidación antes enunciada, refieren a cuatro tópicos fundamentales que contribuyen a esta situación. La presencia del parentesco familiar en el negocio es un plus, dado que genera fuerza de trabajo y consolida al grupo doméstico en torno al comercio. Sumar, que en las viviendas donde operan estos negocios se fusiona el espacio doméstico y productivo y permite sus proyecciones. Consecuencialmente, es posible constatar una cadena de reemplazos familiares entre los operadores de los negocios, una suerte de multifuncionalidad, donde se puede ser el despachador un día y cajero en otro momento, permitiendo mantener los flujos comerciales sin mediar mayores imprevistos. Y también, el que estos emprendimientos se inscriben en una red social, apoyos y solidaridad vecinal. Se advierte que existe una vinculación con las tecnologías de la información digital, pero sobre todo en términos de promoción, comunicación, y difusión de sus actividades a través de las redes sociales y medios de comunicación social a nivel local.

En cuanto a hitos significativos desde la perspectiva de estos emprendedores; estos se remontan al momento de deliberación familiar por echar a andar la actividad económica y que es referido como trascendental de este proceso familiar y comercial. Las motivaciones fueron las necesidades económicas de las familias, y los deseos de autonomía e independencia a estructuras empresariales. El segundo momento, es la operación de estos negocios, donde para sus inicios debieron ejecutarse modificaciones a las estructuras de las viviendas donde se instalaron.

Otro aspecto, es la flexibilidad que debe imperar, dado que estos establecimientos, se adaptan a los horarios vecinales, y sus residentes pueden flanquearlos dado conocimiento de los propietarios, tiempos y ritmos de los negocios. La publicidad sigue los patrones habituales, no obstante, la gran mayoría del vecindario se conocen y saben del establecimiento; y mayoritariamente, se recurre a exhibir los productos a través de publicidad estandarizada de las empresas proveedoras; también imperan medios internos como pizarras y letreros. Los rubros, son posibles agruparlos en tres: almacenes 
de abarrotes; comida rápida y aquellos de ventas de útiles escolares, pero estos límites no son excluyentes.

En las nuevas tecnologías, se aprecia una valoración a estos recursos automatizados que sirven para la función comercial, tanto en el pesaje y conservación de la mercadería, como también máquinas para cortar productos cárneos y hornos eléctricos. La promoción comercial, también es apoyada por Redes Sociales, que son básicamente administradas por los jóvenes de las familias, que ocupan un rol importante en este ámbito. En los negocios, las plataformas gubernamentales son utilizadas, pero básicamente en lo que son consultas de cuestiones tributarias y de Seguridad Social.

Entre los desafíos para fortalecer los negocios de barrio: Promover una cultura de la innovación, para mejores servicios, adaptándose a nuevas necesidades y públicos; proceso sin perder su identidad, que es de los principales valores que distinguen a estos negocios. Capacitación que se ve dificultado por cierta resistencia de parte de los propietarios, que lo reconocen como una necesidad, pero visualizan barreras referidas a escaso tiempo, baja escolaridad o un extenso tiempo sin estudiar, como dicen ellos. También el acceso a tecnologías de la información y la comunicación; ya que se evidencia un uso limitado de estas herramientas.

\section{BIBLIOGRAFÍA}

Gálvez, T., Henríquez, H. y Morales, G. (2009). Holding y Tiendas: La trama que multiplica las ventas y redobla el trabajo. Cuadernos de Investigación 36, Santiago: Dirección del Trabajo.

Lomnitz, L. (1994). Redes sociales, cultura y poder: Ensayos de antropología latinoamericana. México: FLACSO.

Mauss, M. (2009). El ensayo sobre el don. Forma y función del intercambio en las sociedades arcaicas. Argentina: Katz.

Malinowski, B. (2001). Los Argonautas del Pacifico Occidental. Barcelona: Península.

Rothstein, F. (2007). Parentesco y empleo femenino en el México rural: estrategias cambiantes ante el nuevo modelo económico. Familias mexicanas en transición. Unas miradas antropológicas, David Robichaux (coord.). Universidad Iberoamericana. México: 151-184. 